\title{
Construir un multilateralismo favorable al desarrollo: hacia un "nuevo" nuevo orden económico internacional ${ }^{1}$
}

\author{
Ha-Joon Chang
}

\section{Resumen}

En este artículo se señala que el paulatino desplome del orden económico neoliberal internacional, surgido en las décadas de 1980 y 1990, podría estar dejando paso a un "nuevo" nuevo orden económico internacional (NNOEI). En primer lugar, se examina la manera en que el modelo neoliberal se ha debilitado y está siendo replanteado a raíz del declive del sistema comercial internacional multilateral (cuya expresión es la Organización Mundial de Comercio (OMC)) y de la crisis financiera mundial de 2008. También se describe la forma en que los recientes cambios en la economía mundial y en las ideas predominantes, además de una serie de factores contingentes -entre ellos, el cambio climático, el ascenso de China y la crisis de la pandemia de la enfermedad por coronavirus (COVID-19)-, han hecho más probable el surgimiento de un NNOEl, si bien se reconoce que algunos factores podrían obstaculizar el progreso en ese sentido.

\section{Palabras clave}

Desarrollo económico, neoliberalismo, multilateralismo, comercio internacional, OMC, sistemas económicos, crisis económica, COVID-19, países en desarrollo, China

\section{Clasificación JEL}

B27, F02, F55, F63

Autor

Ha-Joon Chang es Director del Centro de Estudios del Desarrollo y Profesor de Economía Política del Desarrollo en la Facultad de Economía de la Universidad de Cambridge. Correo electrónico: ha-joon.chang@econ.cam.ac.uk.

\footnotetext{
Este artículo se basa en la 16 ${ }^{a}$ Cátedra Raúl Prebisch que el autor expuso ante la Comisión Económica para América Latina y el Caribe (CEPAL) el 21 de agosto de 2019. La exposición fue sustancialmente actualizada y ampliada en agosto de 2020, en el contexto de la pandemia del coronavirus (COVID-19) y sus consecuencias. El autor desea expresar su agradecimiento a Alicia Bárcena, Secretaria Ejecutiva de la CEPAL, y a Mario Cimoli, Gabriel Porcile, Miguel Torres y Romain Zivy por sus comentarios sobre la versión preliminar de la disertación.
} 


\section{Introducción}

El orden económico internacional establecido tras la Segunda Guerra Mundial, basado en las instituciones de Bretton Woods, comenzó su declive en la década de 1970. En las décadas de 1980 y 1990, una vez que los programas de ajuste estructural del Fondo Monetario Internacional y el Banco Mundial y el desplome del bloque socialista dieron por terminada la etapa de desarrollismo en el Tercer Mundo, surgió un nuevo orden económico internacional "neoliberal" basado en dejar al mundo entero expuesto a las fuerzas ilimitadas del mercado. Muchos esperaban que este nuevo orden mundial se perpetuaría por mucho tiempo, o quizás para siempre, una idea que quedó plasmada en la expresión "el fin de la historia", que acuñó el científico político estadounidense (entonces) neoconservador Francis Fukuyama (1989).

Sin embargo, el nuevo orden mundial comenzó a perder fuerza lentamente casi inmediatamente después de su surgimiento. El gran entusiasmo por la globalización que generó en 1995 el surgimiento de la Organización Mundial de Comercio (OMC) en reemplazo del Acuerdo General sobre Aranceles Aduaneros y Comercio (GATT), se enfrió rápidamente a raíz de los desacuerdos entre los países ricos y los países en desarrollo durante las conferencias ministeriales posteriores y de las manifestaciones contra la globalización alrededor de las sedes de las conferencias, en particular en Seattle (1999), Cancún (2003) y Hong Kong (2005). A esto pronto le siguió la crisis financiera mundial de 2008, que socavó la confianza en el sistema neoliberal, caracterizado por la apertura de los mercados financieros internacionales. Desde la década de 2010, el ascenso de China, que responde a un orden económico que en esencia difiere del neoliberalismo, si bien no es totalmente incompatible con él, también ha planteado un complejo desafío a la ortodoxia neoliberal. La crisis del coronavirus (COVID-19) en 2020 ha puesto de manifiesto aún más debilidades del sistema neoliberal, y ha obligado a numerosos países a reflexionar sobre la manera en que organizan sus economías y sus sociedades.

En este artículo se examina la cuestión de si el declive del actual modelo económico internacional neoliberal está creando las condiciones idóneas para el surgimiento de un orden alternativo, que denomino un "nuevo" nuevo orden económico internacional (NNOEI), que reemplazaría al nuevo orden económico internacional (NOEI), uno de cuyos principales defensores fue Raúl Prebisch. Este trabajo está estructurado de la siguiente manera. En la sección II se describe el declive del modelo actual de comercio internacional multilateral representado por la OMC. En la sección III se establece la relación que existe entre este declive y las limitaciones del sistema de la OMC. En la sección IV se examinan los factores que podrían obstaculizar el surgimiento de un NNOEI como alternativa. Sin dejar de reconocer la importancia de estos factores, las siguientes secciones están dedicadas al análisis de otros numerosos factores que hacen del surgimiento de un NNOEl una perspectiva probable: cambios en la estructura de la economía mundial (sección V), cambios en las ideas (sección VI) y factores contingentes, como el cambio climático, el ascenso de China y la crisis del COVID-19 (sección VII). En la sección VIII se realizan algunos comentarios a manera de conclusión.

\section{El declive del multilateralismo en el comercio internacional}

En años recientes, y en el contexto de las políticas comerciales agresivas del Gobierno de Trump en los Estados Unidos, ha aumentado la preocupación sobre el futuro del sistema comercial internacional. A fin de contrarrestar lo que desde su punto de vista constituye la competencia "desleal" de China, el Gobierno de Trump ha impuesto aranceles adicionales a las importaciones de ese país. Con el mismo afán, los Estados Unidos han renegociado a su favor tanto el Tratado de Libre Comercio de 
América del Norte (TLCAN) como el acuerdo de libre comercio con la República de Corea. El país ha llegado al extremo de recurrir al uso de aranceles como una manera de reducir los flujos migratorios procedentes de México.

Un rechazo tan flagrante del multilateralismo por parte de los Estados Unidos, en particular en el contexto del aumento del racismo y la xenofobia en los países ricos, ha incrementado la percepción de que el modelo actual de comercio internacional basado en el multilateralismo -es decir, el sistema de la OMC- se enfrenta a una grave amenaza.

Se trata de noticias sumamente negativas para los países en desarrollo. Es un hecho incontrovertible que el multilateralismo ofrece mejores oportunidades para este grupo de países, cuyo poder de negociación individual es escaso frente a los países ricos. El hecho de que los países ricos hayan intentado socavar una y otra vez el multilateralismo cuando va en contra de sus intereses demuestra a las claras que se trata de un modelo que no favorece a los débiles. El mejor ejemplo de esto es la evolución del sistema de la OMC.

Cuando la OMC fue fundada, en 1995, los países ricos expresaron su firme compromiso con el multilateralismo, e incluso llegaron a aceptar el sistema de "un país, un voto" por primera vez en la historia de los principales organismos internacionales. A modo de comparación, los cinco miembros permanentes del Consejo de Seguridad de las Naciones Unidas tienen poder de veto, y las decisiones en el Banco Mundial y en el Fondo Monetario Internacional (FMI) se adoptan esencialmente (si bien existen excepciones) en función del capital que cada país aporta al organismo. Sin embargo, quedó claro que los países ricos apoyaron la idea únicamente porque pensaron que podrían controlar la agenda de negociaciones para influir sobre los países en desarrollo, y que podrían persuadirlos o amenazarlos de manera individual usando estratégicamente los presupuestos bilaterales de ayuda, las vías de acceso a los mercados y la inversión extranjera directa.

Para su sorpresa, los países ricos pronto se dieron cuenta de que no podían intimidar a los países en desarrollo para obligarlos a aceptar cualquier condición que les plantearan en el marco de la OMC. Durante las conferencias ministeriales de Seattle (1999) y Cancún (2003), el predominio de las denominadas "reuniones en la Sala Verde" (de las que solo participaban los países ricos y un número limitado de países en desarrollo que no podían pasarse por alto) hicieron que los países en desarrollo se dieran cuenta de que el multilateralismo prometido por los países ricos era solo de la boca hacia afuera. Como resultado, los países en desarrollo se han opuesto con firmeza a las exigencias irrazonables que los países ricos les han planteado a través de la OMC, por ejemplo, en el proyecto del acuerdo multilateral sobre inversiones o en las negociaciones sobre el acceso a los mercados para los productos no agrícolas.

Cuando los países ricos se percataron de que no podrían hacer lo que querían dentro de la OMC, no dudaron en abandonar sus compromisos con el multilateralismo. Para cuando el Presidente Trump asumió funciones, en los hechos los Estados Unidos ya habían dejado de lado el sistema de la OMC y habían centrado sus esfuerzos en establecer acuerdos comerciales bilaterales y regionales, como el Acuerdo Transpacífico. Por su parte, si bien la Unión Europea no ha rechazado el multilateralismo al mismo grado que los Estados Unidos, sí se ha valido de tácticas del tipo "divide y reinarás" en sus negociaciones comerciales con los países en desarrollo. En 1996, el panel de resolución de controversias de la OMC determinó que la Convención de Lomé, el acuerdo comercial preferencial entre la Unión Europea y los países de África, el Caribe y el Pacífico -que en su mayoría habían sido colonias de los países de la Unión Europea-, era incongruente con el GATT, por lo que la Unión Europea propuso reemplazarlo con un acuerdo comercial y de inversiones denominado el Acuerdo de Asociación Económica. Sin embargo, al pactar el acuerdo, y con el ánimo de debilitar el poder de negociación de ese grupo de países, la Unión Europea deliberadamente los dividió en siete subgrupos regionales en vez de negociar con ellos en conjunto. 
Lisa y llanamente, el sistema comercial multilateral, cuya revitalización iría en beneficio de los países en desarrollo, comenzó a desmoronarse bastante tiempo antes de la llegada de Trump a la Presidencia de los Estados Unidos. Sin embargo, para lograr esa revitalización no alcanza con volver al sistema de la OMC, que necesita de una reforma radical para deshacerse de sus sesgos intrínsecos contra los países en desarrollo y transformarse en una herramienta que verdaderamente promueva el desarrollo. A fin de analizar cuáles son esos sesgos, cómo pueden contrarrestarse y de qué manera se puede avanzar hacia un orden económico internacional más equitativo, en este trabajo se hace referencia a las ideas de Raúl Prebisch, tal como fueron plasmadas en el llamamiento que las Naciones Unidas hicieron en 1974 para el establecimiento de un nuevo orden económico internacional².

\section{El sistema de la OMC y sus limitaciones}

El sistema de la OMC se basa en el principio de que el libre comercio es lo que más conviene a todos los países en casi todas las circunstancias. Sin embargo, las teorías económicas y los antecedentes históricos demuestran que a largo plazo el libre comercio entre países que transitan etapas diferentes del desarrollo económico es perjudicial para las naciones menos desarrolladas. Pese a que en el corto plazo existe la probabilidad (aunque no la certeza) de que el libre comercio permita a todos los socios comerciales maximizar su producción y sus ingresos, a largo plazo perjudica el desarrollo económico de los socios menos desarrollados, para quienes es imposible establecer industrias tecnológicamente avanzadas y de alta productividad si deben competir con los productores más avanzados de los países con mayor desarrollo económico. Eso implica que, a menos que los gobiernos de los países de menor desarrollo económico se valgan de aranceles, subsidios y otras medidas de apoyo para proteger y resguardar a sus industrias incipientes de alta productividad, les resultará imposible propiciar su crecimiento y, con ello, desarrollar sus economías.

Este es el conocido argumento de la "industria naciente", que propuso por primera vez nada menos que el primer Secretario del Tesoro de los Estados Unidos, Alexander Hamilton, en el informe que presentó en 1791 ante el Congreso de los Estados Unidos (Hamilton, 2001). Esta teoría sentó las bases de la política de desarrollo económico de prácticamente todas las naciones que ahora son ricas cuando en su etapa como países en desarrollo intentaban ponerse al nivel de los países económicamente más avanzados. Como han demostrado Bairoch (1993), Chang (2004b y 2007), y Reinert (2007), todos los países que actualmente son ricos, con la excepción de los Países Bajos y (hasta la Primera Guerra Mundial) Suiza, recurrieron al proteccionismo durante la mayor parte del período en que intentaron llegar al nivel de las economías más avanzadas.

El Reino Unido y los Estados Unidos, los supuestos creadores del libre comercio (o al menos es así como se presentan ante el mundo), fueron los países más proteccionistas en sus respectivos períodos de transición, y defendieron a sus industrias de los productores más avanzados de lo que actualmente son Bélgica y los Países Bajos, en el caso del Reino Unido, y de los productores del Reino Unido, Francia y otros países europeos, en el caso de los Estados Unidos. Durante la mayor parte de estos períodos - de mediados del siglo XVIII a mediados del siglo XIX en el caso del Reino Unido, y de mediados del siglo XIX a mediados del siglo XX en el de los Estados Unidos-, las tasas arancelarias industriales promedio de estos países se ubicaron entre un 40\% y un 50\% (véase el cuadro 1).

\footnotetext{
2 Véase Naciones Unidas (1974).
} 


\section{Cuadro 1}

Países ricos seleccionados: tasas arancelarias promedio sobre los productos manufacturados durante sus primeras etapas de desarrollo

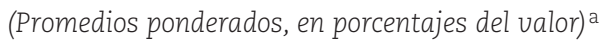

\begin{tabular}{|c|c|c|c|c|c|c|}
\hline & $1820^{b}$ & $1875^{b}$ & 1913 & 1925 & 1931 & 1950 \\
\hline Alemaniac $^{c}$ & 8 a 12 & 4 a 6 & 13 & 20 & 21 & 26 \\
\hline Austriad $^{d}$ & $\mathrm{R}$ & 15 a 20 & 18 & 16 & 24 & 18 \\
\hline Bélgicae $^{e}$ & 6 a 8 & 9 a 10 & 9 & 15 & 14 & 11 \\
\hline Canadá & 5 & 15 & n. d. & 23 & 28 & 17 \\
\hline Dinamarca & 25 a 35 & 15 a 20 & 14 & 10 & n. d. & 3 \\
\hline España & $\mathrm{R}$ & 15 a 20 & 41 & 41 & 63 & n. d. \\
\hline Estados Unidos & 35 a 45 & 40 a 50 & 44 & 37 & 48 & 14 \\
\hline Francia & $\mathrm{R}$ & 12 a 15 & 20 & 21 & 30 & 18 \\
\hline Italia & n. d. & 8 a 10 & 18 & 22 & 46 & 25 \\
\hline Japón ${ }^{\dagger}$ & $\mathrm{R}$ & 5 & 30 & n. d. & n. d. & n. d. \\
\hline Países Bajos ${ }^{e}$ & 6 a 8 & 3 a 5 & 4 & 6 & n. d. & 11 \\
\hline Reino Unido & 45 a 55 & 0 & 0 & 5 & n. d. & 23 \\
\hline Rusia & $\mathrm{R}$ & 15 a 20 & 84 & $\mathrm{R}$ & $\mathrm{R}$ & $\mathrm{R}$ \\
\hline Suecia & $\mathrm{R}$ & 3 a 5 & 20 & 16 & 21 & 9 \\
\hline Suiza & 8 a 12 & 4 a 6 & 9 & 14 & 19 & n. d. \\
\hline
\end{tabular}

Fuente: Elaboración propia, sobre la base de H. J. Chang, Retirar la escalera: la estrategia del desarrollo en perspectiva histórica, Madrid, Los Libros de la Catarata, 2004, P. Bairoch, Economics and World History: Myths and Paradoxes, Hemel Hempstead, Harvester Wheatsheaf, 1993, y K. Taylor, "Tariffs", Encyclopedia of Canada, Vol. VI, W. Stewart Wallace (ed.), Toronto, University Associates of Canada, 1948.

Nota: $\mathrm{R}=\mathrm{Se}$ aplicaban numerosas e importantes restricciones a las importaciones de bienes manufacturados, por lo que las tasas arancelarias medias carecen de relevancia.

a En la publicación del Banco Mundial, World Development Report 1991: The Challenge of Development, Nueva York, Oxford University Press, 1991, pág. 97, recuadro 5, figura un cuadro similar, que en parte hace referencia a los estudios de Bairoch sobre los que se basa el cuadro anterior. Sin embargo, pese a su gran similitud en la mayoría de los casos, el hecho de que las cifras del Banco Mundial sean promedios no ponderados y las de Bairoch promedios ponderados hace de aquellas la opción menos preferible.

b Se trata de índices sumamente aproximados que aportan una serie de promedios, no de valores extremos.

c La cifra de 1820 corresponde únicamente a Prusia.

d Austro-Hungría antes de 1925.

e En 1820, Bélgica y los Países Bajos formaban parte del Reino Unido de los Países Bajos.

f Hasta 1911, el Japón estuvo obligado a mantener tasas arancelarias bajas (como máximo de un 5\%) en virtud de una serie de "tratados desiguales" celebrados con los países europeos y con los Estados Unidos. En el recuadro a que se hace referencia anteriormente (Banco Mundial, 1991), se asignan al Japón tasas arancelarias no ponderadas promedio para todos los bienes (no solo los bienes manufacturados) de un 13\% para 1925, un 19\% para 1930, y un 4\% para 1950.

Aun cuando sus tasas arancelarias promedio no fuesen demasiado altas, muchos de los países ricos de la actualidad brindaban importantes protecciones arancelarias a determinadas industrias estratégicas, además de otras medidas de apoyo. Por ejemplo, si bien sus aranceles industriales promedio se ubicaban entre un $15 \%$ y un $20 \%$ a finales del siglo XIX y principios del siglo XX. Alemania y Suecia aplicaron aranceles mucho más altos a sus industrias pesada y química, sectores emergentes cuyo desarrollo les permitió ponerse al nivel del Reino Unido. Otro ejemplo es Bélgica, cuyas tasas arancelarias industriales promedio a finales del siglo XIX se ubicaban en apenas un 10\%, pero que aplicaba aranceles de entre un $30 \%$ y un $60 \%$ a los textiles y de un $85 \%$ al hierro. Aun en el período que siguió a la Segunda Guerra Mundial, el nivel de proteccionismo siguió siendo alto hasta la década de 1960. Recién para la década de 1970 los países ricos de la actualidad tuvieron aranceles industriales promedio que estaban por debajo de los que aplican los países en desarrollo actualmente, que se ubican en alrededor de un 10\% (véase el cuadro 2). 


\section{Cuadro 2}

Países ricos seleccionados: tasas arancelarias promedio sobre los bienes manufacturados en las primeras etapas del período posterior a la Segunda Guerra Mundial

(En porcentajes)

\begin{tabular}{|c|c|c|c|c|c|}
\hline & 1950 & 1959 & 1962 & 1973 & 1979 \\
\hline \multicolumn{6}{|l|}{ Europa } \\
\hline Bélgica & 11 & 14 & & & \\
\hline Francia & 18 & 30 & & & \\
\hline Alemania Occidental & 26 & 7 & & & \\
\hline Italia & 25 & 18 & & & \\
\hline Países Bajos & 11 & 7 & & & \\
\hline Promedio de la Comunidad Económica Europea (CEE) ${ }^{\mathrm{a}}$ & & 15 & 13 & 8 & 6 \\
\hline Austria & 18 & & $20^{\mathrm{b}}$ & 11 & 8 \\
\hline Dinamarca & 3 & & & & \\
\hline Finlandia & & & $>20^{c}$ & 13 & 11 \\
\hline Suecia & 9 & & 8 & 6 & 5 \\
\hline Japón & n. d. & & 18 & 10 & 6 \\
\hline Reino Unido & 23 & & 16 & & \\
\hline Estados Unidos & 14 & & 13 & 12 & 7 \\
\hline
\end{tabular}

Fuente: Elaboración propia, sobre la base de P. Bairoch, Economics and World History: Myths and Paradoxes, Hemel Hempstead, Harvester Wheatsheaf, 1993; H. Grubel y H. Johnson "Nominal tariffs, indirect taxes and effective rates of protection: the common market countries 1959", The Economic Journal, vol. 77, nro. 308, 1967; B. Balassa, "Tariff protection in industrial countries: an evaluation", Journal of Political Economy, vol. 73, nro. 6, 1965; P. Katzenstein, Small States in World Markets: Industrial Policy in Europe, Ithaca, Cornell University Press, 1985; D. Greenaway, International Trade Policy: from Tariffs to the New Protectionism, Basingstoke, Macmillan Press, 1983, y M. Panić, National Management of the International Economy, Basingstoke, Macmillan Press, 1988.

a En el promedio de la CEE posterior a 1973 se incluye a Dinamarca y el Reino Unido.

b Datos para 1960.

c Estimación del autor. Los datos sobre las tasas arancelarias de Finlandia no son fáciles de conseguir, pero la información consignada en el cuadro 8.2 de Panić (1988) señala que en 1965 los ingresos por concepto de aranceles como porcentaje de todas las importaciones de Finlandia se ubicaron en un 9,97\%, un guarismo considerablemente más alto que el del Japón $(7,55 \%)$ o el de Austria (8,57\%), cuyas tasas arancelarias industriales promedio se ubicaron en un $18 \%$ y un $20 \%$, respectivamente. Con estos datos, no sería ilógico suponer que las tasas arancelarias industriales promedio de Finlandia a mediados de la década de 1960 estaban muy por encima del 20\%.

Por último, pero no menos importante, los países ricos de la actualidad recurrieron a numerosas barreras no arancelarias al comercio, en particular en el período posterior a la Segunda Guerra Mundial; algunas de ellas fueron las prohibiciones a las importaciones, la aplicación de cuotas de importación (por ejemplo, las notorias restricciones voluntarias a las exportaciones que los Estados Unidos y los países europeos impusieron a los fabricantes japoneses de automóviles en las décadas de 1970 y 1980) y las normas para los productos (por ejemplo, requisitos sanitarios y fitosanitarios para los productos alimentarios).

No fue solo en lo referido a las políticas comerciales que los países ricos hicieron todo lo que ahora les dicen a los países desarrollados que no hagan dentro del sistema de la OMC. Sucede lo mismo en ámbitos tales como los subsidios, la inversión extranjera directa y los derechos de propiedad intelectual.

Mediante el Acuerdo sobre Subvenciones y Medidas Compensatorias, el Acuerdo sobre las Medidas en materia de Inversiones relacionadas con el Comercio y el Acuerdo sobre los Aspectos de los Derechos de Propiedad Intelectual Relacionados con el Comercio, la OMC restringe la capacidad de acción de los países en lo referido al uso de subsidios, a la reglamentación de la inversión extranjera directa y a la reducción de la protección de los derechos de propiedad intelectual, todas ellas medidas que los países en desarrollo necesitan más que los países ricos. Sin embargo, cuando los países ricos de la actualidad estaban intentando desarrollar sus propias economías, usaron de manera agresiva los subsidios industriales, establecieron un sinfín de regulaciones para la inversión extranjera directa, y de manera deliberada no protegieron los derechos de propiedad intelectual de los extranjeros (para más información, véase el capítulo 2 de Chang (2004a) y Chang (2004b)). 
Al señalar que las políticas de desarrollo que utilizaron los países ricos de la actualidad van en contra de las normas de la OMC, no estoy diciendo que un sistema de comercio internacional que favorezca el desarrollo necesariamente debe permitir todas las medidas de política que los países ricos aplicaron en el pasado. Por ejemplo, en el siglo XIX, Austria, los Estados Unidos, Francia, los Países Bajos y el Reino Unido permitían a sus ciudadanos registrar patentes de invenciones diseñadas por extranjeros. Dicha práctica no solo sería inaceptable hoy, sino que existen mejores maneras de ayudar a los países en desarrollo a acceder a tecnologías avanzadas, por ejemplo, suspender patentes seleccionadas para invenciones que son esenciales para el desarrollo económico, reducir para los países en desarrollo las regalías que se pagan por concepto de licencias, o impulsar políticas activas de transferencia de tecnologías.

Lo que importa no es la política específica que se aplique, sino el principio que la sustenta: que un sistema multilateral que realmente favorezca el desarrollo debe basarse en el principio de proteccionismo asimétrico. En ese sistema, se permitiría a los países de menor desarrollo económico aplicar más medidas de protección y regulación que los países más ricos, con la expectativa de que, a medida que sus economías se desarrollan para alcanzar el nivel de los países más acaudalados, gradualmente eliminen las políticas adicionales de protección.

En respuesta a esta propuesta, quienes defienden el sistema de la OMC sostienen que sería injusto permitir que en un sistema multilateral las condiciones no sean las mismas para todos. Sin embargo, este argumento es engañoso.

La noción de igualdad de condiciones es un principio esencialmente bueno al cual parece imposible oponerse. Sin embargo, es necesario cuestionarlo si queremos avanzar hacia un orden económico internacional que realmente favorezca el desarrollo.

De más está decir que la igualdad de condiciones es el principio que debe adoptarse cuando todos los participantes son iguales. Por ejemplo, si los equipos nacionales de fútbol del Brasil y de la Argentina se enfrentaran en una Copa del Mundo, sería absolutamente injusto permitir a los brasileños atacar cuesta abajo y obligar a los argentinos a atacar cuesta arriba.

Por otro lado, cuando los participantes no son iguales, obligarlos a competir en pie de igualdad no es lo correcto. Si el equipo nacional de fútbol de Brasil se enfrentase a un equipo de niñas de 10 años, lo justo sería que las niñas atacaran cuesta abajo, y los brasileños cuesta arriba. Por supuesto, en la vida real, las canchas de fútbol no están inclinadas, precisamente porque no permitimos que jugadores desiguales compitan entre sí.

Eso no se limita al fútbol. En todos los deportes está estrictamente prohibido que jugadores desiguales compitan entre sí. En el boxeo, en la lucha libre, en el taekwondo, en el levantamiento de pesas y en muchos otros deportes, existen categorías de peso con límites claramente establecidos; por ejemplo, en el boxeo, las categorías de peso más livianas se diferencian en apenas 2 a 3 libras $(1 \mathrm{~kg}$ a 1,5 kg). En todos los deportes, existen categorías por edad: los equipos de adultos no pueden competir contra equipos infantiles o juveniles. En el golf, existe un sistema específico de hándicaps que permite a los jugadores más débiles competir con ventajas en proporción (inversa) a sus habilidades de juego.

En respuesta a estas críticas, los defensores del sistema de la OMC sostienen que ya existe un mecanismo de trato especial y diferenciado para los países en desarrollo, por lo que no corresponde introducir otras reformas.

Sin embargo, las ventajas que este mecanismo prevé para los países en desarrollo son mínimas. Y si bien los países menos adelantados reciben algunos beneficios adicionales, como el uso de subsidios a las exportaciones, las ayudas son muy pocas. La única medida favorable importante para los países en desarrollo es que se les otorga un poco más de tiempo para aplicar las normas de la OMC (en general, de cinco a ocho años más), pero en última instancia tienen que ceñirse a las normas de la misma manera que los países ricos. 
Lo que es más importante, me atrevería a decir que el "trato especial y diferenciado" no tiene nada de "especial". Aplicar normas distintas a los países en desarrollo no debería considerarse como un trato especial, como tampoco lo son las rampas para quienes usan sillas de ruedas o la escritura en braille para los ciegos. Se trata simplemente de un trato diferenciado para países distintos con necesidades y capacidades diferentes.

En pocas palabras, un multilateralismo que verdaderamente favorezca el desarrollo debe crear el mayor "espacio de política" posible en materia de políticas para que los países apliquen políticas adaptadas a sus propias capacidades y necesidades. En este sentido, se necesita una versión actualizada del NOEI que propuso Raúl Prebisch; podríamos denominarla un "nuevo" nuevo orden económico internacional, o NNOEl.

\section{IV. ¿Hacia un NNOEI?}

Mi llamamiento a instaurar un NNOEI probablemente sea considerado como un proyecto destinado al fracaso, al igual que sucedió cuando en las décadas de 1970 y 1980 se promovió el establecimiento de un NOEl. De hecho, podría decirse que las perspectivas de lograr una reforma del orden económico internacional son hoy todavía más débiles.

En primer lugar, la sensación de culpa poscolonialista que muchas personas de los países ricos sentían en la década de 1970 y que en cierto modo impulsó el proyecto para un NOEl, ha perdido intensidad en el último medio siglo.

En segundo lugar, ya no existe una competencia sistémica entre el bloque capitalista y el bloque socialista, una situación que dio cierto poder de negociación a los países en desarrollo que pudieron ubicarse a ambos lados del conflicto, como sucedió en la India.

En tercer lugar, el rechazo contra un NOEl en el periodo neoliberal ha hecho que algunas ideas que figuran en la propuesta sean muy difíciles de llevar a cabo. Por ejemplo, el Acuerdo sobre las Medidas en materia de Inversiones relacionadas con el Comercio ha hecho difícil revitalizar la idea de exigir que las corporaciones transnacionales se ciñan a un código de conducta, lo que era un aspecto importante de la propuesta para un NOEl.

Por último, pero no por ello menos importante, el predominio que ha adquirido la ideología neoliberal en decenios recientes, pese a los golpes que han supuesto para ella acontecimientos recientes como la crisis financiera mundial de 2008 y la crisis del COVID-19, ha hecho que los países en desarrollo se muestren más dispuestos a aceptar el actual orden económico internacional neoliberal.

Sin embargo, existen algunos factores que van en contra de dicha tendencia, que pueden dividirse en tres grupos: cambios en la estructura de la economía mundial, cambios en las ideas y factores contingentes. Los factores se examinan con mayor detalle a continuación.

\section{Cambios en la economía mundial}

En primer lugar, los países en desarrollo ahora tienen mucho más peso en el sistema económico mundial que cuando se presentó la propuesta para un NOEl, en gran medida, si bien no exclusivamente, como resultado del ascenso de China. En 1974, los países ricos representaban casi cuatro quintos de la economía mundial (4,189 billones de dólares de un total de 5,312 billones de dólares). Para 2018, representaban menos de dos tercios (54,108 billones de dólares de un total de 85,791 billones de dólares).

La crisis del COVID-19 probablemente acelere esta transición en la participación de los países ricos y los países en desarrollo en la economía mundial. Según las proyecciones del FMl para finales 
de junio de 2020, este año las economías avanzadas se contraerán un 8\%, frente a apenas un 3\% en el caso de los mercados emergentes y las economías en desarrollo (FMl, 2020a). Además, se prevé que en 2021, la recuperación de los países en desarrollo será del orden del 5,9\%, mientras que la proyección de crecimiento para las economías avanzadas es de apenas un 4,8\%. Sin embargo, al momento de escribir este artículo (mediados de agosto de 2020), menos de dos meses tras la publicación de las proyecciones del FMI, esas estimaciones suenan demasiado optimistas, en particular para los países ricos. Incluso si las proyecciones no experimentaran modificaciones, eso implicaría que, a finales de 2021, el producto de los países en desarrollo sería un 2,7\% más alto que en 2019, mientras que el de los países ricos estaría un 3,6\% por debajo, lo que supone un cambio importante en sus participaciones relativas en la economía mundial. Por cuanto las recesiones en los países ricos, que serán más profundas, probablemente tengan efectos de histéresis más graves en sus economías que en los países en desarrollo, donde los efectos serán más leves, cabe esperar que el crecimiento relativo de los países ricos sea aún más bajo en los próximos años que lo que fue antes de 2020.

En segundo lugar, las interacciones entre los países en desarrollo han cobrado una importancia sin precedentes. El comercio Sur-Sur ha aumentado sustancialmente y pasó de representar un promedio del 41,8\% del comercio mundial entre 1995 y 1997 a un promedio del 57,4\% entre 2015 y 2017. Además, ese aumento no se debió únicamente al ascenso de China, como algunos podrían pensar. Sin incluir a China, el comercio Sur-Sur pasó de un 34,8\% del comercio mundial en el período transcurrido entre 1995 y 1997 a un 42,1\% para el período comprendido entre 2015 y 2017 (FMl, 2020b). Además, China, la India y otros países en desarrollo se han transformado en importantes actores financieros de la economía internacional en lo referido a la concesión de préstamos, la ayuda exterior y la inversión extranjera directa. Más recientemente, han surgido nuevas instituciones financieras multilaterales dirigidas por el Sur, como el Nuevo Banco de Desarrollo y el Banco Asiático de Inversión en Infraestructura. Por ende, los países en desarrollo han reducido su dependencia de los países ricos, y con ello pueden ejercer una mayor presión para el establecimiento de un NNOEl.

\section{Cambios en las ideas}

Además de los cambios en la economía mundial, los cambios que se han producido en las ideas durante los últimos 50 años aumentarán las probabilidades de avanzar hacia un NNOEI. En primer lugar, las ideas favorables al desarrollo que se consideraban demasiado radicales en los días del NOEI ahora son aceptables, precisamente debido al legado histórico del NOEl, y a cierto grado han sido puestas en práctica. Por ejemplo, algunos países económicamente avanzados ya han alcanzado y superado la meta de que la asistencia oficial para el desarrollo equivalga a un 0,7\% del PIB, que se consideró muy optimista en 1970, cuando el compromiso se plasmó en la resolución 2626(XXV) de la Asamblea General de las Naciones Unidas. Otro ejemplo es la condonación de las deudas de los países en desarrollo, algo que muchos consideraban poco realista pero que se ha transformado en una realidad gracias la Iniciativa para los Países Pobres muy Endeudados de 2012. Lo que es más importante, la adopción del sistema de "un país, un voto" en una institución multilateral, que hace 50 años se consideraba apenas un sueño, constituye uno de los fundamentos del sistema de la OMC, pese a que no ha funcionado muy bien en la práctica, como ya se analizó.

En segundo lugar, la crisis financiera mundial de 2008 redujo fundamentalmente la confianza en la eficacia de los mercados financieros abiertos y no regulados, uno de los aspectos fundamentales del orden económico internacional neoliberal. Si bien debido a la fuerte resistencia del sector financiero los sistemas financieros neoliberales no han experimentado reformas profundas desde 2008, ni a nivel nacional ni mundial, las ideas neoliberales que sustentan dichos sistemas ahora gozan de una aceptación mucho más reducida que antes. Además, desde la crisis, los países ricos han recurrido a 
políticas monetarias, como la aplicación de tasas de interés extremadamente bajas y la flexibilización cuantitativa, que en esencia contradicen los principios neoliberales. Habida cuenta de que los principios monetaristas de una política monetaria restrictiva y tipos de interés elevados se consideraban esenciales para propiciar el entorno macroeconómico necesario para la existencia de mercados financieros no regulados y eficientes, la adopción de tales políticas ha socavado aún más los fundamentos teóricos para el sistema financiero neoliberal.

Por último, pero no menos importante, la crisis del COVID-19 ha echado por tierra gran parte de la sabiduría convencional del neoliberalismo con respecto al papel del gobierno. Pese a la prédica neoliberal contra la participación del gobierno en la propiedad y la gestión de las empresas, gobiernos de todo el mundo han nacionalizado de jure y de facto algunas empresas clave del sector privado, y están subsidiando directa o indirectamente a muchas otras a una escala gigantesca. En muchos países ricos, los gobiernos han estado preservando el empleo durante los confinamientos impuestos para frenar la pandemia del COVID-19, para lo que se han hecho cargo del pago de hasta un $80 \%$ de los salarios para mantener a los trabajadores en la plantilla, sin importar que estén o no trabajando, o que lo hagan solo a tiempo parcial. Muchos gobiernos, aun aquellos que están ideológicamente en contra de pagar un ingreso de origen público para apoyar a los pobres, como sucede con el Gobierno de Trump en los Estados Unidos, han aumentado los beneficios por desempleo y los subsidios a los ingresos, con varios niveles de cobertura. Gobiernos de todo el mundo están acumulando enormes déficits presupuestales y han abandonado la doctrina del equilibrio presupuestal a la que se venían aferrando -si no en los hechos, sí en las palabras- desde la crisis financiera de 2008. Un ejemplo particularmente llamativo es el del Gobierno de Alemania, conocido por su conservadurismo fiscal, que abolió la ley que establecía un límite máximo a la deuda pública para poder gestionar la crisis del COVID-19 con mayor eficacia valiéndose de herramientas presupuestales. Como se ve, prácticamente todos los principios de la ortodoxia neoliberal han sido abandonados o radicalmente modificados, lo que abre la perspectiva para un discurso político muy distinto en los próximos años.

\section{Factores contingentes}

El tercer elemento que puede propiciar el surgimiento de un NNOEl son los factores contingentes que pueden favorecer a los países en desarrollo, y cuando digo "contingentes" no me refiero a que sean menos importantes.

Uno de los factores es el cambio climático, que está alcanzando un punto de inflexión. La urgencia del problema está obligando a la humanidad a darse cuenta de que el destino será el mismo para todos, lo que está aumentando la presión sobre los países ricos para que hagan más por los países en desarrollo y los ayuden a enfrentar la crisis, en particular mediante transferencias de tecnología a gran escala para mitigar el cambio climático y adaptarse a sus efectos. De hecho, la enorme injusticia de que los países en desarrollo son quienes corren el mayor riesgo de sufrir las peores consecuencias del cambio climático -por ejemplo, más tormentas y sequías y el aumento del nivel del mar-, a pesar de que casi no han contribuido al problema, implica que adoptar un enfoque más equitativo a nivel mundial frente a esta cuestión es una obligación moral, no una mera necesidad técnica.

Otro factor es el ascenso económico de China, una nación que ocupa la posición inédita de ser uno de los principales actores en la economía mundial y, a la vez, un país en desarrollo. Esto afectará la evolución del orden económico internacional. Mientras que en 1974 la economía china representaba apenas un 2,7\% de la economía mundial (144.000 millones de dólares de un total de 5,312 billones de dólares), en 2018 representó un 16\% (13,9 billones de dólares de un total de 86,4 billones de dólares) 3 .

3 Datos del Banco Mundial. 
Dado que los efectos de la pandemia del COVID-19 sobre la economía china han sido mucho menores que en las economías ricas, China adquirirá todavía más protagonismo en la economía mundial.

Aunque no puede decirse que China necesariamente sea un promotor de los intereses colectivos de los países en desarrollo, y pese al grado de dependencia que existe entre China, en cuanto nación manufacturera, y otros países en desarrollo, que la abastecen con materias primas, es innegable que la singularidad de su condición como una de las principales economías del mundo y, a la vez, un país en desarrollo, hace que exista un marcado contraste entre su comportamiento y la de otras economías importantes, como queda claro al observar sus enfoques en materia de prestación de ayuda, inversión extranjera directa y desarrollo de infraestructuras. Además, con China como potencial prestamista, inversor y socio comercial, los países en desarrollo tienen un mayor poder de negociación en sus transacciones con los países ricos y las instituciones financieras multilaterales que estos dominan.

Por último, la crisis del COVID-19 tendrá dos consecuencias que afectarán la organización y la gestión de la economía mundial en los próximos años. En primer lugar, la pandemia ha aumentado la conciencia sobre el hecho de que la humanidad comparte el mismo destino, una situación que el cambio climático ha dejado a la vista de todos, por cuanto se trata de la primera pandemia realmente mundial. En segundo lugar, la crisis del COVID-19 está cambiando la opinión de los países en desarrollo sobre los países ricos.

Al momento de escribir este trabajo, el fracaso de algunos de los países más ricos del mundo en el manejo de la pandemia ha dejado un saldo de cientos de miles de muertes evitables, mientras que algunas de las sociedades más pobres, como Etiopía, Rwanda, Viet Nam y el estado de Kerala, en la India, se han enfrentado a la crisis con un éxito destacable, a pesar de los escasos recursos de que disponen. Por otro lado, las tasas de mortalidad del COVID-19 en los Estados Unidos, Francia y el Reino Unido, países que alardeaban de ser los guardianes de la civilización y que periódicamente sermonean a otros países sobre la importancia de la buena gobernanza, de la eficacia del gobierno (si es pequeño) y de los derechos humanos, han sufrido (y siguen sufriendo) la pérdida de cientos de miles de vidas como consecuencia de errores de gestión, de la incompetencia, del caos social y, por sobre todas las cosas, de su flagrante menosprecio por los derechos humanos, en particular los de los pobres, las personas mayores y las minorías.

A raíz de ello, al compararse con los países europeos o con aquellos con poblaciones mayoritariamente europeas, muchos países en desarrollo han comenzado a cuestionar su sensación de inferioridad frente a los países ricos, una percepción que tiene su origen en una historia de siglos y siglos de colonialismo, imperialismo, dominación económica y adoctrinamiento cultural. Una vez que este cambio de perspectiva se arraigue, los países en desarrollo dejarán de titubear frente a los países ricos con el temor, la admiración y el respeto de antaño. Esto modificará la dinámica de la política internacional, y ayudará a los países en desarrollo a ser más firmes en sus negociaciones con los países ricos.

\section{Observaciones finales}

Los países en desarrollo deben oponerse a los intentos de los países ricos de socavar el multilateralismo. No obstante, no deben limitarse a intentar restaurar la versión neoliberal del multilateralismo que encarna la OMC. El orden económico internacional debe reformarse de manera tal que sea más favorable al desarrollo. En otras palabras, es necesario instaurar un "nuevo" nuevo orden económico internacional basado en el reconocimiento de que el sistema económico internacional debe maximizar el espacio político para que países distintos con necesidades y capacidades distintas puedan adoptar las políticas económicas que mejor se ajusten a su situación.

Al igual que en las décadas de 1970 y 1980, cuando las circunstancias paralizaron el progreso hacia un NOEl, hoy existen factores que obstaculizarán el avance hacia un NNOEl, entre otros, la 
gradual desaparición de la sensación de culpa poscolonial en los países ricos, la eliminación de la competencia sistémica entre el bloque capitalista y el bloque socialista, los mecanismos adoptados como parte de la ofensiva contra el establecimiento de un NOEl durante el periodo neoliberal, y el predominio de la ideología neoliberal.

Con todo, existen otros factores que podrían facilitar el establecimiento de un NNOEl. En primer lugar, los cambios que se han producido en la estructura de la economía mundial, como la creciente importancia de los países en desarrollo en la economía global y la profundización de las interacciones económicas entre ellos, han mejorado las probabilidades para el surgimiento de un NNOEI. En segundo lugar, han surgido nuevas ideas que favorecen la noción de un NNOEI, entre ellas: la aceptación generalizada de algunos de los principios favorables al desarrollo que se exponen en la propuesta para un NNOEI (en particular en lo referido a la ayuda exterior, a la condonación de las deudas y a la estructura de votación de los organismos internacionales); tras la crisis financiera mundial de 2008, el rechazo de la idea de que los mercados financieros abiertos y no regulados son más eficientes; y el abandono de la ortodoxia neoliberal en lo referido al papel general del Estado en el contexto de la crisis del COVID-19. Por último, pero no menos importante, existen factores contingentes que podrían propiciar el surgimiento de un NNOEl, entre otros, la mayor conciencia sobre el hecho de que la humanidad comparte el mismo destino frente a desafíos como el cambio climático y la pandemia de COVID-19; el ascenso de China, un país en desarrollo, como una de las principales economías del mundo, y el hecho de que los países en desarrollo están superando su complejo de inferioridad frente a los países ricos, en particular al constatar los errores en que muchos de ellos han incurrido al enfrentarse a la crisis del COVID-19.

Por supuesto, llevará tiempo consolidar un NNOEl, si es que alguna vez surge. Por ende, en el ínterin los países en desarrollo deberán aprender a manejarse en un sistema que no se caracteriza por la equidad. Pese a la opinión popular, es mucho lo que puede hacerse en este sentido, pero eso es una cuestión que trasciende el alcance de este trabajo ${ }^{4}$.

\section{Bibliografía}

Bairoch, P. (1993), Economics and World History: Myths and Paradoxes, Hemel Hempstead, Harvester Wheatheaf. Banco Mundial (1991), World Development Report 1991: The Challenge of Development, Nueva York, Oxford University Press.

CEPA (Comisión Económica para África) (2016), Transformative Industrial Policy for Africa, Addis Abeba.

Chang, H. J. (2007), ¿Qué fue del buen samaritano? Naciones ricas, políticas pobres, Madrid, Intermón Oxfam Editorial.

(2004a), "Regulation of foreign investment in historical perspective", The European Journal of Development Research, vol. 16, № 3, septiembre.

(2004b), Retirar la escalera: la estrategia del desarrollo en perspectiva histórica, Madrid, Los Libros de la Catarata.

FMI (Fondo Monetario Internacional) (2020a), "Una crisis como ninguna otra, una recuperación incierta", Actualización de las Perspectivas de la Economía Mundial, Washington, D.C., junio.

(2020b), "Direction of Trade Statistics" [base de datos en línea] https://data.imf.org/?sk=9D6028D4F14A-464C-A2F2-59B2CD424B85.

Fukuyama, F. (1989), "The end of history?", The National Interest, No. 16, Center for the National Interest.

Hamilton, A. (2001), "Report on the subject of manufactures, December 5, 1791", Hamilton: Writings, Nueva York, The Library of America.

Naciones Unidas (1974), "Declaración sobre el establecimiento de un nuevo orden económico internacional" (A/RES/3201(S-VI)) [en línea] https://undocs.org/es/A/RES/3201(S-VI).

Reinert, E. (2007), La globalización de la pobreza: cómo se enriquecieron los países ricos... y por qué los países pobres siguen siendo pobres, Libros de Historia, Barcelona, Crítica.

4 Véase un análisis más exhaustivo en el capítulo 5 de CEPA (2016). 